\title{
ASSOCIATION BETWEEN LONELINESS AND SUICIDALITY DURING MIDDLE CHILDHOOD AND ADOLESCENCE: LONGITUDINAL EFFECTS AND THE ROLE OF DEMOGRAPHIC CHARACTERISTICS
}

\author{
A thesis submitted \\ to Kent State University in partial \\ fulfillment of the requirements for the \\ degree of Master of Arts.
}

by

Katherine C. Schinka

August, 2011 
Thesis written by

Katherine C. Schinka

B.A., Queens University of Charlotte, 2008

M.A., Kent State University, 2011

\section{Approved by}

Manfred H. M. van Dulmen, Ph.D. Advisor

Maria S. Zaragoza, Ph.D.

Chair, Department of Psychology

Timothy Moerland, Ph.D

Dean, College of Arts and Sciences 


\section{TABLE OF CONTENTS}

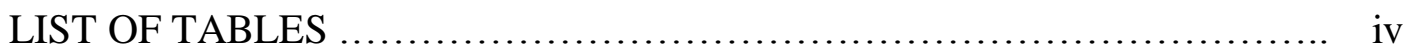

INTRODUCTION ......................................................... $\quad 1$

METHOD ............................................................. 10

Participants ...................................................... 10

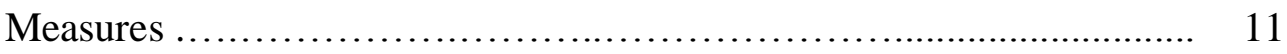

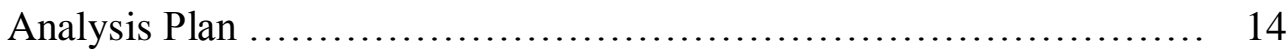

RESULTS ......................................................... 16

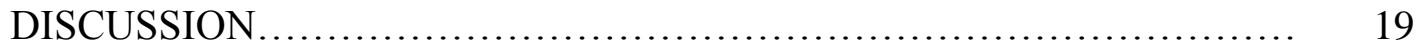

REFERENCES....................................................... 25 


\section{LIST OF TABLES}

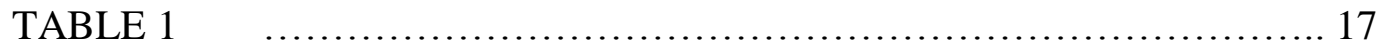

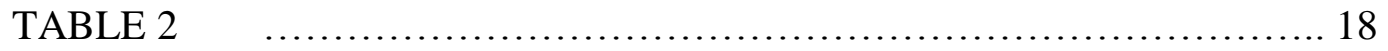




\section{INTRODUCTION}

Suicide is the third leading cause of death in youth between the ages of 10 and 14 , resulting in more than 4000 adolescent deaths per year (Centers for Disease Control and Prevention (CDC), 2008). Suicide represents an important health crisis around the world, and research is continually needed to help us understand and prevent suicide. Researchers and psychologists most commonly use demographic characteristics to understand and predict suicidality (e.g. CDC, 2008; Joiner, 2005). This research provides much needed knowledge about certain groups, such as minorities or low income people, who might be more at risk for suicide. Recently, however, the trend in suicide research has been to focus on psychosocial factors by themselves and in conjunction with demographic factors (e.g. Rutter \& Behrendt, 2004). This study aims to investigate one important psychosocial risk factor for suicide, loneliness, across different demographic characteristics both crosssectionally and longitudinally.

\section{Suicide and Demographic Characteristics}

Gender. Gender differences in suicide rates are well documented (for a review, see Smalley, Scourfield, \& Greenland, 2005). In fact, the gender difference in suicide "is one of the most empirically documented social facts in suicidology" (Stack \& Wasserman, 2009; p. 13). Although females attempt suicide and deliberately self-harm at 
three times the rate of males, males die by suicide at four times the rate of females (CDC, 2008; McMahan, Reulbach, Corcoran, Keeley, Perry, \& Arensman, 2010). Therefore, males represent over three-quarters of all suicides in the United States (CDC, 2008).

This "gender paradox" (Canetto \& Sakinofsky, 1998) occurs almost universally, and it does not seem to be an artifact of data collection methods. Instead, the choice of method of attempt contributes most to this gender discrepancy. Males tend to choose more lethal methods of suicide attempt, including firearm use. Females, on the other hand, use less lethal methods, such as poisoning (CDC, 2008). Method differences are attributed to either (1) intent, with females exhibiting less intent to actually die, and (2) bodily disfigurement, with females less likely to want to alter their appearance in any way (Stack \& Wasserman, 2009).

Ethnicity. There are clear differences in rates of suicide across ethnic groups. Across all ages, American Indian/Alaskan Native males have the highest suicide rates with Caucasian males falling close behind (CDC, 2008). Lower suicide rates in adult minority populations have been attributed to increased social support and religious connections in certain subpopulations, such as Hispanics (Joiner, 2005). Strong ethnic identity has also been related to lower rates of suicide attempts (Cheng et al., 2010). There is some evidence that the same mechanism may be at work in adolescents. In a clinical sample, African American adolescents reported more social support than Caucasian participants, and those adolescents who reported less social support also had higher depression scores and reported more suicidal ideation (Joe, Clarke, Ivey, Kerr, \& 
King, 2007). Unfortunately, this study is one of the few that empirically support the notion that strong social ties prevent suicide in minority populations.

In fact, in the past decade, minority suicide rates (Hispanics, African Americans, Native Americans) have increased (Rutter \& Behrendt, 2004). African American adolescents have a significantly higher attempt rate than Caucasian adolescents, and African American males have experienced the greatest rate increase in suicidality in recent decades (Joe \& Marcus, 2003). Furthermore, African American adolescents have both the highest number and percentage of suicides in all of the African American population, and cohort studies have shown them to be more at-risk than older age-groups (Crosby \& Molock, 2006; Joe, 2006).

Socioeconomic Status. Suicide is often associated with socioeconomic status through economic strain. Economic strains may include job loss, demotion, loss of home, or receiving government assistance (Rojas \& Stenberg, 2010; Stack \& Wasserman, 2007). One qualitative study, for instance, found that medical records of suicide victims indicated unemployment almost half of the time (Stack \& Wasserman, 2007). In addition, Chinese researchers found an inverse association between economic growth and suicide rates for both males and females (Zhang, et al., 2010). As gross domestic product and average income increased, suicide rates decreased.

Although very few studies have investigated the relationship between economic strain and suicide in adolescents, it is likely that an association does exist and that young people can be impacted by economic strain either directly or through intergenerational factors. In one such study, Rojas and Stenberg (2010) used data from 7,719 men in the 
Stockholm Birth Cohort. They found that poverty during childhood — as defined by receiving government assistance — was associated with increased risk for suicide between the ages of 17 and 30 years old.

Another study on economic strain in adolescents investigated 622 high school students living in a rural Midwestern farming community (Lempers, Clark-Lempers, Simone, 1989). Lempers and colleagues used the Economic Hardship Questionnaire to measure income loss and changes in lifestyle caused by economic hardship and the Beck Depression Inventory as a measure of distress. They found that economic strain in the family is directly linked to distress.

\section{$\underline{\text { Suicide and Psychosocial Risk }}$}

Overall, there is a clear pattern to how demographic factors influence suicidality. Although females have a higher suicide attempt rate, males die by suicide at a higher rate than females. As far as ethnicity is concerned, minority adolescents have a higher suicide rate than Caucasian adolescents. Lastly, lower income adolescents or those who experience economic strain in their families tend to have higher suicide rates. These three demographic variables are helpful in determining which groups may be more at risk for suicidality.

However, identifying psychosocial risk factors in addition to and in conjunction with demographic characteristics may be more informative in pinpointing youth at risk. An adolescent who is lonely and a member of a demographic group that is more at risk 
(e.g. a female, a minority, a low-income adolescent) may have a higher probability of experiencing suicidal thoughts or behaviors. Further, psychosocial factors are more useful in tailoring prevention and intervention programs. Psychosocial factors, as opposed to demographic factors, are modifiable, so at-risk children and adolescents can be identified and interventions can be implemented.

Problems in interpersonal relationships are often cited as psychosocial risk factors for suicide. This is particularly true during adolescence, when romantic relationship dissolution is cited as a primary precipitating factor of suicide (American Association of Suicidology, 2009). Further, the Interpersonal-Psychological Model of Suicide posits that thwarted belongingness, or a feeling of disconnection from peers and family, is one of three major factors leading to suicide (Joiner, 2005). Loneliness can represent thwarted belongingness, and it has often been associated with suicidality in adult and adolescent samples (e.g. Heinrich \& Gullone, 2005).

\section{Loneliness in Middle Childhood and Adolescence}

Loneliness results from the perception that one's interpersonal relationships do not meet personal expectations and thus are deficient (Peplau \& Perlman, 1982). Although loneliness is considered a normal part of development, it can cause considerable distress. Loneliness interferes with an innate human need for belongingness in social groups (Heinrich \& Gullone, 2006; Joiner, 2005). 
Adolescents tend to experience loneliness more intensely than other age groups, with some studies concluding that loneliness peaks in adolescence (Brennan, 1982; Hawthorne, 2008; Goossens, 2006; van Roekel, Scholte, Verhagen, Goossens, \& Engels, 2010). A recent study of a large sample of adolescents from the NICHD Study of Early Child Care found that $36.1 \%$ of participants experienced increases in loneliness from middle childhood into adolescence (Schinka, van Dulmen, Mata, Swahn \& Bossarte, 2011). Adolescents likely report high levels of loneliness for two reasons. First, as young people enter the teenage years, peers become an increasingly important form of social support and their needs for intimacy, attachment, and acceptance are met more by peers than by family (Goossens, 2006; Parkhurst \& Hopmeyer, 1999). In addition, some adolescents start spending more time alone, and they tend to perceive aloneness as negative instead of a time for quiet solitude (Koenig \& Abrams, 1999).

Loneliness has consistently been associated with negative mental health outcomes. Adolescents who suffer from chronic loneliness are more likely to experience more psychopathology, including internalizing and externalizing problems (Goossens, 2006; Koenig \& Abrams, 1999; Schinka, van Dulmen, Mata, Swahn, \& Bossarte, 2011). I recently found that children and adolescents who experience chronically high levels of loneliness across middle childhood and adolescence are more likely to report depression, suicidality, aggression, and social skills deficits in adolescence (Schinka, van Dulmen, Mata, Swahn, \& Bossarte, 2011). Further, children who perceive themselves to become lonelier throughout middle childhood and adolescence display more depression and externalizing problems compared to those children who do not become lonelier over time 
(Jones, Schinka, van Dulmen, Bossarte, \& Swahn, in press). Evidence that loneliness predicts depression even when controlling for baseline depression symptoms further corroborates this link (Koenig \& Abrams, 1999).

\section{$\underline{\text { Loneliness and Suicide }}$}

Loneliness can be severe enough to lead to death by suicide (Heinrich \& Gullone, 2006; Trout, 1980). In general, the perception of a lack of satisfying interpersonal relationships can be so damaging to adolescents that it predicts suicidality (Jeglic, Pepper, Vanderhoff, \& Ryabchenko, 2007). One cross-sectional school study of over 8,000 Thai, Taiwanese, and Filipino adolescents found a positive association between loneliness and suicide (Page, Yanagishita, Suwanteerangkul, Zarco, Mei-Lee, \& Maio, 2006). Using the UCLA Loneliness Scale, these researches found a significant association between loneliness and number of suicide attempts in the past 12 months and between loneliness and lifetime suicide attempts. Further, Roberts and colleagues (1998) found that adolescents with a high degree of loneliness were two times as likely to exhibit suicidal ideation.

A study by Negron and colleagues (1997) helps to parse out the details in how loneliness differentially predicts suicidal ideation and suicide attempts. They compared 35 suicidal ideators and 32 suicide attempters between the ages of 12 and 17 who had been admitted to an emergency room because of suicidal crisis. The researchers used semi-structured interviews to investigate the amount and intensity of stressful life events 
and emotional problems in the adolescents' lives prior to admittance to the hospital. The researchers found that suicide attempters were more likely to report more isolation as compared to suicide ideators. However, the two groups did not differ on demographic characteristics or depressive symptoms during their suicide crisis. Although this study was retrospective and used only a clinical sample, it shows that isolation may play an important role in differentiating adolescents who attempt suicide versus those adolescents who only think about it.

Summary

The above studies clearly delineate an association between suicide and demographic factors and suicide and loneliness. The implications of these associations have significant shortcomings that limit their generalizability to children and adolescents. First, many studies concerning demographic factors, specifically socioeconomic status, are limited by using adult-only samples. The implications for risk in children and adolescents are largely unknown. Second, studying demographic factors is important for epidemiological purposes because it is informative about who is at risk, but it does not inform us why these subgroups may be at risk (Rutter \& Behrendt, 2004). Few studies on psychosocial risk factors use separate analyses across demographic groups (i.e. separately for males and females; e.g. Page, Yanagishita, Suwanteerangkul, Zarco, Mei-Lee, \& Maio, 2006), or test the interactions between psychosocial and demographic risk. Finally, the research on loneliness and suicide is limited by cross-sectional designs. More 
research is needed to see if loneliness impacts suicidality over the long-term. The current research aims to investigate the effect of loneliness on suicidality and how demographic factors may act to moderate this relationship. Studying demographic factors and loneliness in conjunction may provide a more complete picture for who is at risk for suicide; this may prove helpful in tailoring inventions for youth at risk.

\section{Goals of the Current Study}

The current study has three goals. First, I want to see if the effect of loneliness on suicidality is time-limited (cross-sectional) or longitudinal (from middle childhood to adolescence). Second, I will test whether loneliness predicts suicidality above and beyond demographic characteristics. Third, I will explore how ethnicity, gender, and socioeconomic status may moderate the link between loneliness and suicidality. In addition, I will explore these aims separately for suicidal thoughts and suicidal behaviors since previous studies indicate psychosocial risk factors may differentially predict them (Negron, Piacentini, Graae, Davies, \& Shaffer, 1997). Based on previous research (e.g. Page, Yanagishita, Suwanteerangkul, Zarco, Mei-Lee, \& Maio, 2006; Roberts, Roberts, \& Chen, 1998), I expect that loneliness will be concurrently associated with suicidal ideation and suicidal behavior. Although it has not been previously tested, I expect this association to also hold longitudinally. Since little empirical evidence exists to indicate a moderating effect of demographic factors on the loneliness and suicide link, these analyses will primarily be exploratory. 


\section{METHOD}

\section{$\underline{\text { Participants }}$}

Data for this study came from 1364 study participants in the NICHD Study of Early Child Care (NICHD SECC, NICHD Early Child Care Research Network, 2000). The NICHD SECC is a national, multi-site study that followed children from birth through age 15 and gathered information during four phases. Phase one data was collected between birth and age three; phase two data collection occurred between age three and grade two; phase three data collection occurred during grades three through seven; phase four data collection took place at age 15. During each phase, data was collected from multiple informants, including mothers, fathers, after-school caregivers, teachers, and the study children themselves.

The current analyses used data from 937 participants with complete data on all study variables in phases three and four of this dataset. The current sample is almost equally distributed among genders (50.4\% male, $49.6 \%$ female). Participants identified themselves in the following ethnic groups: $77.1 \%$ Caucasian, $11.8 \%$ Black, $5.9 \%$ Hispanic, $1.3 \%$ Asian, and $4.0 \%$ Other.

Based on my inclusion criteria, 427 participants were excluded from the current

analyses. These participants lacked data on the loneliness variable (20.9\%), the suicidal ideation items $(27.3 \%)$, suicidal attempt items $(27.3 \%)$, or the income-to-needs ratio 
variable $(27.0 \%)$. Since gender and ethnicity information is collected at birth, all participants had data on these variables.

I conducted attrition analyses to see if the current sample differed from the those who were part of the NICHD SECC but not included in these analyses $(\mathrm{N}=427)$ on any demographic variables, including gender, ethnicity, socioeconomic status. A chi-square statistic was used to investigate gender differences between the included and not included groups. AVOVAs were used to look for differences in ethnicity and socioeconomic status between these groups. Results indicated no statistically significant differences on any of these variables between the included and excluded participants.

\section{$\underline{\text { Measures }}$}

Demographic Characteristics. Gender, ethnicity, and socioeconomic status were the included demographic variables. Ethnicity was categorized into two groups: White and non-White. Socioeconomic status was measured using an income-to-needs ratio. The income-to-needs ratio variable was created by the NICHD SECC using an equation reflecting total family income and the number of persons in the family. Higher incometo-needs ratios indicate higher affluence. Income-to-needs ratios lower than one indicate families living below poverty line.

Loneliness. Loneliness was measured at grades three and five and at age 15 using the Loneliness and Social Dissatisfaction Questionnaire (Asher, Hymel, \& Renshaw, 1984; see Appendix A for complete measure). Sixteen items from this self-report 
measure examine the study child's perception of his/her peer relationships. Item examples include "I feel alone," "I don't have anyone to play with," "There's nobody I can go to when I need help," and "I'm lonely." Each item was scored on a Likert scale from one ("not at all true") to five ("always true"). Higher scores indicate higher levels of loneliness. In middle childhood, the highest loneliness score, whether it be in grade three or five, was used for each study child. The correlation between the scores in these two grades was moderate $(\mathrm{r}=.41, \mathrm{p}<.001)$.

The Loneliness and Social Dissatisfaction Questionnaire has been shown to be a psychometrically sound measure. The internal consistency for this measure was high at grade three $(\alpha=.87)$, grade five $(\alpha=.91)$, and at age $15(\alpha=.91)$. In addition, it has been tested with African American and Hispanic-American children and has been found to be a psychometrically valid measure for use in minority populations (Bagner, Storch, \& Roberti, 2004). Factor analysis of the measure items yielded a single underlying loneliness factor that included all 16 items (Asher, Hymel, \& Renshaw, 1984). Further, Asher and colleagues (1984) found total loneliness score to be moderately associated with peer-rated sociometric status, such that higher loneliness scores on the Loneliness and Social Dissatisfaction Questionnaire were related to lower sociometric ratings. Although loneliness is a unique construct, this finding suggests that it is related to other measures of social functioning.

Suicidal Ideation. Suicidal ideation was measured at age 15 using parent report from The Child Behavior Checklist and the Youth Self-Report (Achenbach, 1991/1992). Items included "Child talks about killing self" (CBCL) and "I think about killing myself" 
(YSR). Item responses were dichotomized into "yes" and "no" responses. Endorsement of either of these items by the parent or study youth constituted suicidal ideation. Based on parental report, 24 participants endorsed suicidal ideation. Based on youth report, 45 participants endorsed suicidal ideation. Thus, at age $15,7.4 \%$ reported suicidal thoughts $(\mathrm{N}=69)$.

Suicidal Behavior. Suicidal behavior was measured at age 15 using mother report from the Child Behavior Checklist and the Youth Self-Report (Achenbach, 1991/1992). Items included "Child harms self/attempts suicide" (CBCL) and "I deliberately try to hurt or kill myself" (YSR). Item responses were dichotomized into "yes" and "no" responses. Similar to the suicidal ideation construct, endorsement of either item from either informant constituted suicidal behavior. Based on parental report, 19 participants endorsed suicidal behavior. Based on youth report, 19 participants endorsed suicidal behavior. Together, $4.1 \%$ of the current sample reported suicidal behavior at age 15 $(\mathrm{N}=38)$.

While suicidal ideation is often a strong predictor suicidal behavior (Lewinsohn, Rohde, and Seeley, 1994), these two variables do not necessarily coexist. In the current sample, 17 participants endorsed both the suicidal ideation and suicidal behavior items. The remaining participants endorsed only the item for suicidal ideation or the item for suicidal behavior. 


\section{$\underline{\text { Analysis Plan }}$}

Binary logistic regressions were used in this study because dichotomous group membership (suicidal behavior or no suicidal behavior; suicidal ideation or no suicidal ideation) was predicted from a continuous independent variable. The suicidal ideation and suicidal behavior variables were both dichotomized such that zero indicated "no" to suicidal ideation or suicidal behavior and one indicated "yes" to suicidal ideation or suicidal behavior. Suicidal behavior and suicidal ideation were investigated separately as previous research suggests differences in the risk factors for each (Negron, Piacentini, Graae, Davis, \& Shaffer, 1997).

Several sets of logistic regression analyses were conducted. The first model tested the cross-sectional link between loneliness and suicidal ideation at age 15 . The second model tested the cross-sectional link between loneliness and suicidal behaviors at age 15 . The next two logistic regressions tested these same relationships, but longitudinally instead of cross-sectionally. I tested the link between loneliness in middle childhood and suicidal ideation at age 15 and the link between loneliness in middle childhood and suicidal behaviors at age 15 . In all of the above analyses, ethnicity, gender, and socioeconomic status were included as control variables.

The next set of logistic regression analyses included additional interaction terms to investigate whether demographic variables (ethnicity, gender, income-to-needs ratio) moderated the association between loneliness and suicidal ideation/behaviors. To create these interaction terms, I multiplied the standardized loneliness score by (1) the 
dichotmized ethnicity variable, (2) gender, and (3) the standardized income-to-needs ratio. 


\section{RESULTS}

Table 1 contains the bivariate associations for the predictor variables with suicidal ideation and suicidal behavior. Independent sample t-tests were used for the continuous predictor variables (i.e. income, middle childhood loneliness, and adolescent loneliness). Chi-square analyses were used for categorical variables (i.e. sex and ethnicity). Bivariate analyses indicated a statistically significant relationship between adolescent loneliness and suicidal ideation and adolescent loneliness and suicidal behavior.

In the current sample, the mean loneliness score during middle childhood was $30.53(S D=9.63)$ and the mean loneliness score at age 15 was $26.41(S D=8.67)$. In the current study, there were no statistically significant differences between males and females on loneliness during middle childhood (male $M=30.96$; female $M=30.46$; $t(935)=2.10, p>.05)$ and at age 15 (male $M=26.88$; female $M=25.97 ; t(935)=1.06$, $p>.05)$.

I first wanted to establish whether loneliness was cross-sectionally associated with suicidal behavior and thoughts after controlling for demographic factors (see Table 2). The first model indicated that loneliness at age 15 was concurrently related to suicidal thoughts at age $15\left(\mathrm{e}^{\mathrm{B}}=1.94, p<.001\right)$. The second model indicated that adolescent loneliness at age 15 was also concurrently related to suicidal behavior at age $15\left(\mathrm{e}^{\mathrm{B}}=1.86\right.$, $\mathrm{p}<.001)$. In other words, risk for suicidal thoughts and suicidal behavior increased by $94 \%$ and $86 \%$ respectively for each one point increase in loneliness at age 15 . 
Table 1. Bivariate associations for the study variables $(N=937)$

\begin{tabular}{lcccc}
\hline & $\begin{array}{c}\text { Suicidal } \\
\text { Ideation }\end{array}$ & Test & $\begin{array}{c}\text { Suicide } \\
\text { Behavior }\end{array}$ & Test \\
\hline \% Males/females & $3.1 / 3.8$ & $X^{2}(1)=.93$ & $1.8 / 2.1$ & $X^{2}(1)=.30$ \\
\% White/Other & $5.7 / 1.3$ & $X^{2}(1)=.78$ & $3.0 / 1.0$ & $X^{2}(1)=.05$ \\
Mean (SD) Income & $3.73(2.52)$ & $t(935)=3.62$ & $4.82(5.04)$ & $\mathrm{t}(935)=3.28$ \\
Mean (SD) MC Lone & $32.64(9.98)$ & $t(935)=.43$ & $33.34(10.46)$ & $\mathrm{t}(935)=.36$ \\
Mean (SD) Adol Lone & $33.00(10.53)$ & $t(935)=8.20^{* *}$ & $32.84(12.46)$ & $\mathrm{t}(935)=13.07 * *$ \\
\hline
\end{tabular}

Note. $* p<.05 * * p<.001$

MC Lone $=$ Middle childhood loneliness; Adol Lone $=$ age 15 loneliness

Next, I investigated whether the effect of loneliness on suicidal behavior and thoughts extends across time (see Table 2). These analyses found that higher levels of loneliness in middle childhood were associated with suicidal behavior at age $15\left(\mathrm{e}^{\mathrm{B}}\right.$ $=1.36, p<.05$ ), suggesting that with each one point increase in loneliness in middle childhood, risk for suicidal behavior at age 15 increased by $36 \%$. Loneliness is middle childhood, however, was not significantly associated with suicidal thoughts at age 15 .

A second set of analyses was run separately using the different demographic variables -- gender, ethnicity, and income-to-needs ratio -- as moderators. None of these analyses produced statistically significant results. These statistics indicate that gender, ethnicity, and socioeconomic status do not moderate the association between loneliness and suicidal ideation or the association between loneliness and suicidal behavior. 
Table 2. Results of Logistic Regression Analyses Predicting Adolescent Suicidal Behavior and Suicidal Ideation from Loneliness during Middle Childhood and Adolescence $(N=937)$

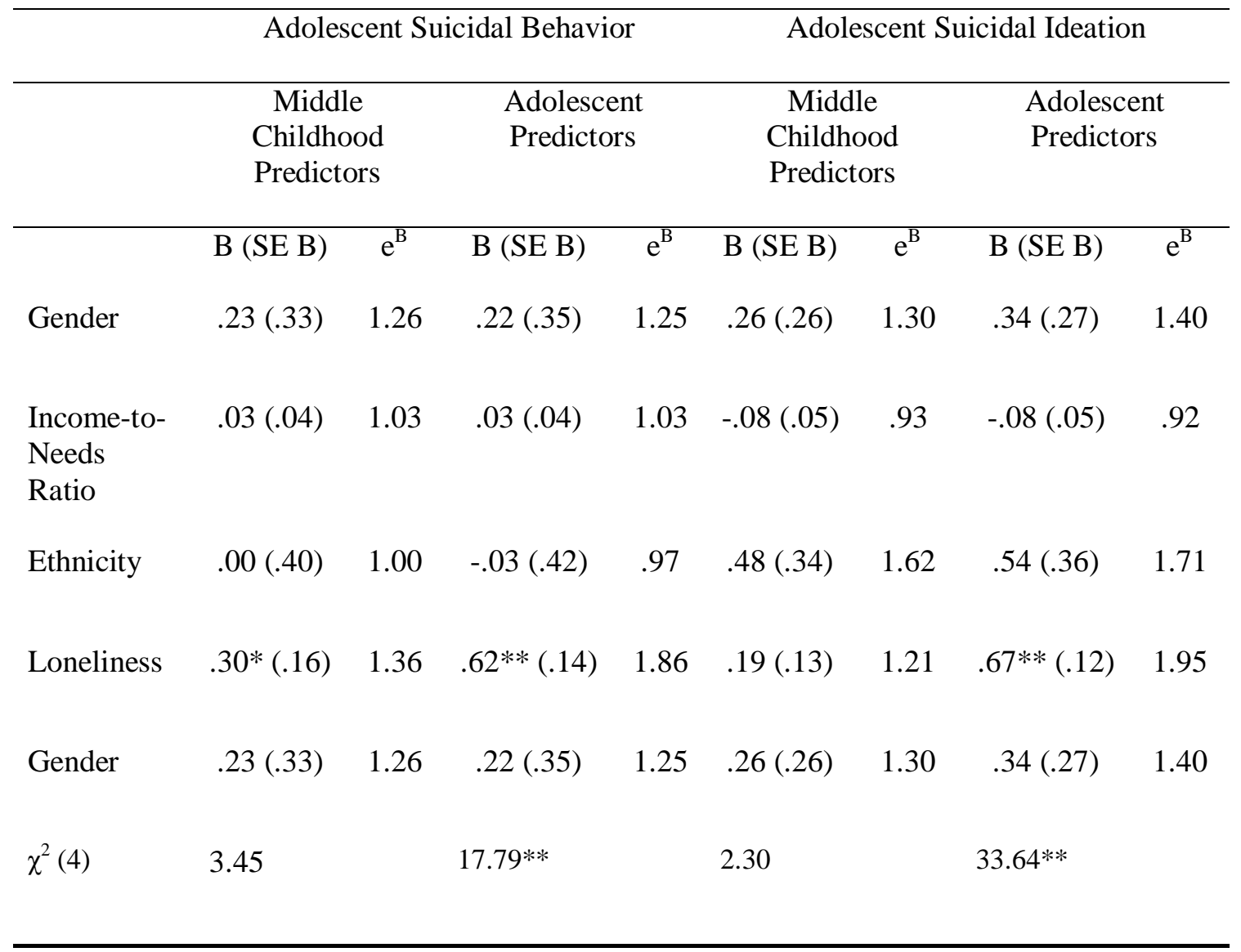

Note. ${ }^{*} \mathrm{p}<.05,{ }^{* * \mathrm{p}}<.001$ 


\section{DISCUSSION}

The purpose of the current study was to investigate whether loneliness in middle childhood and adolescence is related to suicidal thoughts and suicidal behaviors crosssectionally and longitudinally. In addition, this study investigated differences in these connections among different demographic factors, namely gender, ethnicity, and socioeconomic status. I found loneliness to be related to suicidality. Specifically, this study found a longitudinal link between loneliness in middle childhood and suicidal behaviors at age 15 . However, the link between loneliness and suicidal thoughts did not hold up across time and was only cross-sectional in nature. Further, there were no differences in the association between loneliness and suicidality among different demographic groups. Ethnicity, gender, and socioeconomic status did not moderate the association between loneliness and suicidality.

The findings in the current study are consistent with previous research on loneliness and suicide (Heinrich \& Gullone, 2006) and also offer several important extensions. First, the loneliness and suicide link has primarily been studied in adult populations. The current study extended this finding to children and adolescents by showing both concurrent and longitudinal associations between loneliness and suicidality. Extending this association to children and adolescents is important because loneliness is likely more prevalent during the non-adult years (Heinrich \& Gullone, 2006). 
Hawthorne (2008) found participants between the ages of 15 years and 30 years to be specifically at risk for loneliness. In a study of second, fourth, and sixth graders, Luftig (1987) found that the younger children reported the highest levels of loneliness. The current results suggest that loneliness is not just a temporary problem of childhood but rather may be a serious interpersonal issue with long-term consequences.

Second, my findings add to the research by suggesting a longitudinal element to the link between loneliness and suicidal behavior. Past studies have been limited by cross-sectional designs that do not allow prediction across time. Loneliness does impact immediate psychological functioning, as shown through the association between age 15 loneliness and age 15 suicidal thoughts and suicidal behaviors. However, the current study is important because it shows that loneliness may also have serious long-term effects on psychological functioning. Namely, loneliness in middle childhood longitudinally impacted suicidal behavior at age 15 .

Third, previous studies focused on suicidality as either a broad construct or as consisting of only suicidal thoughts or suicidal behaviors. This study eliminated this drawback by differentiating between suicidal ideation, or thoughts about suicide, and suicidal behaviors. This differentiation is important since Negron and colleagues (1997) found differences in social isolation between suicide attempters and suicide ideators. This distinction was salient since the current study found a longitudinal link with only suicidal behaviors and not suicidal thoughts. Given the lack of specificity in longitudinal research in this area, this finding is important as it raises the question of how predictors of suicidal 
thoughts and behaviors differ longitudinally. Perhaps psychosocial risk factors are important as longitudinal predictors for suicidal behaviors only. Further research is needed to understand why longitudinal risk may differ for suicidal thoughts versus behaviors.

Fourth, a large proportion of suicide research has been based solely on demographic factors, and studies using only minority populations have been mostly qualitative in nature or have only reported on prevalence rates (e.g. Joe, 2006; Joe \& Marcus, 2003). The current study built off these studies in two ways. First, I investigated whether a psychosocial variable - loneliness - predicted suicidality above and beyond demographic factors. Second, I studied how demographic factors in conjunction with loneliness predicted suicidality. According to the current results, loneliness did predict suicidality when demographic variables were controlled for, and demographic factors did not moderate the relationship between loneliness and suicidal thoughts or suicidal behaviors. This finding shows that, although demographic characteristics are informative about prevalence rates, psychosocial factors are important in predicting suicidality and should be the focus of prevention efforts.

\section{Implications for Practice}

The results of current study serve as further evidence that demographic factors alone cannot tell us who is at risk for suicidal thoughts and behaviors. Neither gender, ethnicity, nor socioeconomic status were associated with suicidal ideation or suicidal 
behavior in adolescence. In addition, these demographic characteristics did not moderate the relationship between loneliness and suicidality. These results suggest that interventions should not be tailored based on demographic characteristics. Instead, psychosocial factors - specifically those concerning loneliness - are important for understanding suicidality. In this case, loneliness impacted both concurrent and future suicidality.

The longitudinal association between loneliness and suicidal behavior is particularly meaningful in that it shows how perceived deficits in interpersonal relationships may have serious long-term effects. This knowledge informs approaches to intervention by emphasizing the impact of social dissatisfaction in childhood on longterm psychological functioning. Intervention should be focused on children who report loneliness or those who perceive that they do not have anyone to play with or talk to.

\section{Limitations of the Current Study}

Suicide is a low base rate phenomenon, and the data in this study are no exception. The current national rate of suicidal ideation is $13.8 \%$ and the rate of suicide attempt is $6.3 \%$ (Youth Risk Behavior Survey; Eaton, et al., 2010). As a community lowrisk sample, the current sample's rates of suicidal ideation and behavior were lower than national averages $-7.4 \%$ and $4.1 \%$, respectively. In addition, it is possible that the null demographic results were the product of a fairly high functioning, homogeneous sample. 
The sample was mostly Caucasian with only a small percentage of families living on the threshold of poverty.

Further, valid measurement of loneliness is often debated. Researchers have argued that loneliness should be investigated in a multidimensional way, or by researching multiple informants, multiple contexts, and different affinities for loneliness (Marcoen, Goossens, \& Caes, 1987). The Loneliness and Social Dissatisfaction Questionnaire (Asher, Hymel, \& Renshaw, 1984) is a unidimensional measure since it reflects loneliness only in the peer context. Thus, I can only generalize these findings and the important relationship between loneliness and suicidality to peer contexts. Future research on the link between loneliness and suicide would be remiss not to include measures of loneliness in several contexts. In particular, it would be interesting to investigate to what extent loneliness in family contexts contribute to mental health functioning.

Finally, the current study assessed loneliness as a lone risk factor for the emergence of suicidality. The development of psychopathology in adolescence is likely influenced by multiple variables (Cicchetti, 2002); thus it has been suggested that suicide may be the result of an interaction of multiple risk factors (eg. Joiner, 2005). It is possible that there are third variables at work that I did not include in this study and that may contribute to the loneliness-suicide relationship. For instance, mental health problems, such as depression or other internalizing problems, may contribute to the perception that one is lonely. Investigating these other mental health variables as potential mediators might further advance our understanding of the loneliness-suicide link. Studying these 
potential other variables would be informative about why some lonely children become suicidal and others do not.

\section{$\underline{\text { Conclusions }}$}

In conclusion, this study illustrated the importance of studying how psychosocial factors, specifically loneliness, are associated with suicidality. Loneliness at age 15 was concurrently associated with suicidal thoughts and suicidal behavior at age 15 , and loneliness in middle childhood was longitudinally associated with suicidal behaviors at age 15. This is one of the first studies to link loneliness in childhood with suicidal behavior in adolescence, thus establishing a longitudinal association. Further, this study found that this association did not differ across ethnicity, gender, or socioeconomic status. 


\section{REFERENCES}

Achenbach, T. M. (1991). Manual for the Youth Self-Report and 1991 profile.

Burlington, VT: University of Vermont, Department of Psychiatry.

Achenbach, T.M. (1992). Manual for the Child Behavior Checklist / 2-3 and 1992

Profile. Burlington, VT: University of Vermont, Department of Psychiatry.

American Association of Suicidology (2009). School Suicide Prevention Accreditation

Manual.

Asher, S. R., Hymel, S., \& Renshaw, P. (1984). Loneliness in children. Child

Development, 55, 1456-1464.

Bagner, D. M., Storch, E. A., \& Roberti, J. W. (2004). A factor analytic study of the Loneliness and Social Dissatisfaction Scale in sample of African-American and Hispanic-American children. Child Psychiatry \& Human Development, 34, 237250.

Brennan, T. (1982). Loneliness at adolescence. In L. A. Peplau \& D. Perlman (Eds.), Loneliness: A sourcebook of current theory, research, and therapy (pp. 269 - 290). New York: Wiley.

Canetto, S. S. \& Sakinofsky, I. (1998). The gender paradox in suicide. Suicide and LifeThreatening Behavior, 28, 1- 23.

Centers for Disease Control and Prevention, National Center for Injury Prevention and 
Control. (2008). Suicide: Facts at a Glance. Retrieved January 4, 2010 from Centers for Disease Control and Prevention, National Center for Injury Prevention and Control Website http://www.cdc.gov/ncipc/dvp/Suicide/SuicideDataSheet.pdf.

Cheng, J. K. Y., Fancher, T. L., Ratanasen, M., Conner, K. R., Duberstein, P. R., Sue, S., \& Takeuchi, D. (2010). Lifetime suicidal ideation and suicide attempts in Asian Americans. Asian American Journal of Psychology, 1, 18 - 30.

Cicchetti, D. \& Rogosch, F. A. (2002). A developmental psychopathology perspective on adolescence. Journal of Consulting and Clinical Psychology, 70, 6-20.

Crosby, A. E. \& Molock, S. D. (2006). Introduction: Suicidal behaviors in the African American community. Journal of Black Psychology, 32, 253-261.

Eaton, D. K., Kann, L., Kinchen, S., Shanklin, S., Ross, J., Hawkins, J., Harris, W. A., Lowry, R., McManus, T., Chyen, D., Lim, C., Whittle, L., Brener, N. D., \& Wechsler, H. (2010). Youth Risk Behavior Surveillance - United States, 2009, MMWR Weekly, 59 (SS 5), 1 - 36.

Goossens, L. (2006). Affect, emotion, and loneliness in adolescence. In S. Jackson \& L. Goossens (Eds.), Handbook of adolescent development (pp. 51 - 70). Hove: Psychology Press.

Hawthorne, G. (2008). Perceived social isolation in a community sample: Its prevalence and correlates with aspects of peoples' lives. Social Psychiatry and Psychiatric Epidemiology, 43, $140-150$. 
Heinrich, L. M. \& Gullone, E. (2006). The clinical significance of loneliness. Clinical Psychology Review, 26, 695 - 718.

Jeglic, E. L., Pepper, C. M., Vanderhoff, H. A., \& Ryabchenko, K. A. (2007). An analysis of suicidal ideation in a college sample. Archives of Suicide Research, 11, $41-56$.

Joe, S. (2006). Explaining changes in the patterns of Black suicide in the United States from1981 to 2002: An age, cohort, and period analysis. Journal of Black Psychology, 32, 262-284.

Joe, S., Clarke, J., Ivey, A . Z., Kerr, D., \& King, C. A. (2007). Impact of familial factors and psychopathology on suicidality among African American adolescents. Journal of Human Behavior in the Social Environment, 15, 199-218.

Joe, S. \& Marcus, S. C. (2003). Datapoints: Trends by race and gender in suicide attempts among U.S. adolescents, 1991-2001. Psychiatric Services, 54, 454.

Joiner, T. E. (2005). Why People Die By Suicide. Cambridge: Harvard University Press.

Jones, A. C., Schinka, K. C., van Dulmen, M.H.M, Bossarte, R., \& Swahn, M. (in press). Changes in loneliness during middle childhood predict risk for adolescent suicidality indirectly through mental health problems. Journal of Clinical Child and Adolescent Psychology.

Koenig, L. J. \& Abrams, R. F. (1999). Adolescent loneliness and adjustment: A focus on gender differences. In K. J. Rotenberg \& S. Hymel (Eds.), Loneliness in childhood and adolescence (pp. 296 - 322). Cambridge: Cambridge University Press. 
Lempers, J. D., Clark-Lempers, D., \& Simons, R. L. (1989). Economic hardship, parenting, and distress in adolescence. Child Development, 60, 25 -39.

Lewinsohn, P. M., Rohde, P., \& Seeley, J.R. (1994). Psychosocial risk factors for future adolescent suicide attempts. Journal of Consulting and Clinical Psychology, 62, $297-305$.

Luftig, R. L. (1987). Children's loneliness, perceived ease in making friends and estimates social adequacy: Development and social metacognition. Child Study Journal, 17, $35-53$.

Marcoen, A., Goossens, L., \& Caes, P. (1987). Loneliness in pre- through late adolescence: Exploring the contributions of a multidimensional approach. Journal of Youth and Adolescence, 16, 561 - 577.

McMahon, E. M., Reulback, U., Corcoran, P., Keeley, H. S., Perry, I. J., \& Arensman, E. (2010). Factors associated with deliberate self-harm among Irish adolescents. Psychological Medicine: A Journal of Research in Psychiatry and the Allied Sciences, 40, 1811-1819.

Negron, R., Piacentini, J., Graae, F., Davis, M., \& Shaffer, D. (1997). Microanalysis of adolescent suicide attempters and ideators during the acute suicidal episode. Journal of the American Academy of Child and Adolescent Psychiatry, 36, 1512 1519.

NICHD Early Child Care Research Network. (2000). The relation of child care to cognitive and language development. Child Development, 71, 958-978.

Page, R. M., Yanagishita, J., Suwanteerangkul, J., Zarco, E. P., Mei-Lee, C., \& Miao, N. 
(2006). Hopelessness and loneliness among suicide attempters in school-based samples of Taiwanese, Philippine, and Thai adolescents. School Psychology International, 27, 583 - 598.

Parkhurst, J. T. \& Hopmeyer, A. (1999). Developmental change in the sources of loneliness in childhood and adolescence: Constructing a theoretical model. In K. J. Rotenberg \& S. Hymel (Eds.), Loneliness in childhood and adolescence (pp. 56 - 79). Cambridge: Cambridge University Press.

Peplau, L. A. \& Perlman, D. (1982). Loneliness: A sourcebook of current theory, research, and therapy. New York: Wiley.

Roberts, R. E., Roberts, C. R., \& Chen, R. Y. (1998). Suicidal thinking among adolescents with a history of attempted suicide. Journal of the American Academy of Child \& Adolescent Psychiatry, 37, 1294-1300.

Rojas, Y. \& Stenberg, S-A. (2010). Early life circumstances and male suicide -- A 30year follow-up of a Stockholm cohort born in 1953. Social Sciences and Medicine, 70, 420 - 427.

Rutter, P. A. \& Behrendt, A. E. (2004). Adolescent suicide risk: Four psychosocial factors. Adolescence, 39, 295-302.

Schinka, K. C., van Dulmen, M. H. M., Mata, A. D., Swahn, M., \& Bossarte, R. (2011, April). Predictors and outcomes of loneliness trajectories from middle childhood to adolescence. Accepted to the Society for Research on Child Development, Montreal.

Smalley, N., Scourfield, J., \& Greenland, K. (2005). Young people, gender, and suicide: 
A review of research on the social context. Journal of Social Work, 5, 133 - 154.

Stack, S. \& Wasserman, I. (2007). Economic strain and suicide risk: A qualitative analysis. Suicide and Life-Threatening Behavior, 37, 103 - 112.

Stack, S., \&\& Wasserman, I. (2009). Gender and suicide risk: The role of wound site. Suicide and Life-Threatening Behavior, 39, 13 - 20.

Trout, D. (1980). The role of social isolation in suicide. Suicide and Life-Threatening Behavior, 10, 10 - 23.

van Roekel, E., Scholte, R. H. J., Verhagen, M., Goossens, L., \& Engels, R.C.M.E (2010). Loneliness in adolescence: Gene x environment interactions involving the serotonin transporter gene. The Journal of Child Psychology and Psychiatry, 51, $747-754$.

Zhang, J., Ma, J., Jia, C., Sun, J., Guo, X., Xu, A., \& Li, W. (2010). Economic growth and suicide rate changes: A case in China from 1982 to 2005. European Psychiatry, 25, $159-163$. 